\title{
离子液体支载反应研究进展
}

\author{
霍聪德* 王 程 \\ (西北师范大学化学化工学院 兰州 730070)
}

\begin{abstract}
摘要 利用离子液体作为可溶性支载体近年来已经发展成为有机合成中的一个重要策略. 底物、催化剂或反应试剂均 可支载到离子液体上. 离子液体支载合成的后处理一般都比较简单. 离子液体支载的催化剂或反应试剂大多可以被回 收重复使用. 本文对离子液体支载反应的研究新进展进行了综述.
\end{abstract}

关键词 离子液体支载; 回收; 循环使用

\section{Progress in Ionic Liquids Supported Reactions}

\author{
Huo, Congde* Wang, Cheng \\ (College of Chemistry and Chemical Engineering, Northwest Normal University, Lanzhou 730070)
}

\begin{abstract}
Using ionic liquids as soluble supports has emerged in recent years as a feasible strategy for organic synthesis. Substrates, catalysts or reagents can all be immobilized onto ionic liquids. The workup of ionic liquids supported synthesis is usually simple. On the other hand, ionic liquids supported catalysts and reagents can often be recovered and reused. The new progress in ionic liquids supported reactions is reviewed.
\end{abstract}

Keywords ionic liquids supported; recycle; recovery

通过支载的方式进行有机合成起源于 1963 年美国 洛克菲勒研究所的 Merrifield 发明的固相多肽合成方法 (SPPS, 获得 1984 年诺贝尔化学奖) ${ }^{[1]}$. 载体为不溶性固 体高分子树脂, 反应完成后固载产物可以容易的从反应 混合物中过滤分离出来. 如今生物高分子的固相合成已 经自动化，可被常规应用于多肽和聚核苷酸合成.

然而固相合成由于其非均相反应本质, 导致: (1)反 应活性低. 例如在多肽合成中通常需要加入大大过量的 氨基酸单体作为反应试剂才能使反应进行完全. 当需要 某种自然界并不大量存在的氨基酸时, 价格相当昂贵; (2)上载率低. 规模化合成时载体价格昂贵, 在药物寡肽 的合成中, 载体的费用加上消耗的过量单体试剂可占到 整个原材料花费的 $60 \%$ 以上. (3)合成过程中无法采用常 规的分析方法, 难以跟踪和检测反应进程.

基于上述固相合成的局限性, 进一步出现了通过高 分子支载的 “液相合成” 多肽的方法, 使用聚乙二醇 (PEGs)等可溶性高分子作为载体, 从而恢复了均相反应

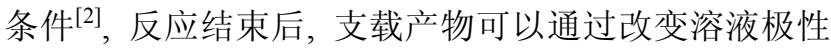
的方式从反应混合物中沉淀分离. 这种合成方式在一定 程度上结合了传统的均相反应条件和固载反应的优点.

上世纪末，基于含氟化合物优先溶解于含氟溶剂的 事实, 氟化物支载合成也有了一定发展, 通过含氟溶剂 萃取等方法, 支载有氟化物的物质可以从反应混合物中 分离出来, 从而使后处理简化 ${ }^{[3]}$.

随着绿色化学和可持续发展化学的概念逐渐深入 人心, 对于离子液体化学的研究全面展开. 1992 年, 美 国南弗罗里达大学的 Zaworotko 小组 ${ }^{[4]}$ 报道了第一例对 空气和水稳定的咪唑阳离子离子液体 ([emim] $\left.\left[\mathrm{BF}_{4}\right]\right)$; 1999 年, 美国南阿拉巴马大学的 Davis 小组 ${ }^{[5]}$ 首次示范 了官能团化的离子液体不仅可以用作反应媒介, 也可用 作反应催化剂, 这篇文章在一定程度上也可以看作是离 子液体支载化合物作为催化剂使用的倠形. 2001 年, 法 国雷恩第一大学的 Bazureau 小组 ${ }^{[6]}$ 首次使用离子液体作 为可溶性载体应用于有机小分子合成. 此后在 21 世纪

\footnotetext{
*E-mail: huocongde@nwnu.edu.cn

Received March 18, 2013; revised April 24, 2013; published online May 9, 2013.

Project supported by the National Natural Science Foundation of China (No. 21002080) and the Specialized Research Fund for the Doctoral Program of Higher Education of the Education Ministry (No. 20106203120003).

国家自然科学基金(No. 21002080)和教育部博士点基金新教师类课题(No. 20106203120003)资助项目.
} 
的第一个十年里, 离子液体载体在有机合成中的应用已 经取得了初步的成果, 在支载催化剂, 反应试剂, 反应 底物方面都取得了不同程度的进展, 开始引起越来越多 有机化学工作者的兴趣，逾百篇相关科技论文已经被发 表 ${ }^{[7]}$.

离子液体作为载体的应用通常首先是离子液体被 官能团化(安装 linker), 从而可以通过共价键与催化剂、 反应试剂或反应底物连接起来. 这种共价键附着的方式 必须足够强壮, 在所要经历的整个反应过程中不会分 解, 但在反应完成后, 又可以方便的切断, 切断的反应 条件不会影响到目标产物. 目前作为离子液体载体的主 力是铵盐离子液体和磷盐离子液体, 特别是杂环铵盐离 子液体, 例如咪唑阳离子液体和吡啶阳离子液体的支载 应用最为广泛, 因为他们在很多化学转化过程中都能够 保持稳定.

离子液体载体可以支载催化剂、反应试剂, 从而方 便回收再利用; 也可以支载反应底物, 用于有机小分子 或生物大分子的多步合成. 由于其离子化特性, 离子液 体支载化合物通常不溶于非极性或小极性有机溶剂(例 如烷烃、醚). 可溶于离子液体或极性有机溶剂(例如乙 腈、 $N, N$-二甲基甲酰胺). 而在中等极性的有机溶剂(例 如氯仿、二氯甲烷)中的溶解性和离子液体载体的结构 和反离子的结构有关.

就目前离子液体支载合成领域发展的情况而言, 离 子液体支载的催化剂大多在离子液体中使用, 反应完成 后, 通过有机溶剂萃取的方式提取产物, 催化剂保留在 离子液体中, 从而可以方便再次使用; 离子液体支载的 有机小分子或生物大分子合成则通常在极性有机溶剂 中进行, 反应完成后, 加入小极性溶剂, 使缚有离子液 体载体的产物从反应液中沉淀出来进行分离.

本文着重论述了进入 21 世纪以来利用离子液体支 载所进行的液相有机合成, 特别侧重于一些相对比较系 统或深入的研究工作.

\section{1 离子液体支载的有机小分子合成}

Bazureau 小组 ${ }^{[8]}$ 在 2001 年首次使用咪唑型离子液 体作为载体应用于有机小分子合成, 他们开发了一系列 具有短的聚乙二醇链的离子液体载体, 通过酯化反应支 载反应底物, 并将其应用于一系列杂环化合物的合成 (Scheme 1).

2003 年, 美国纽约州立大学宾汉姆顿分校的 Handy 小组 ${ }^{[9]}$ 从可再生资源-果糖出发, 4 步反应合成了一系列 新型咪唑类离子液体载体. 这类咪唑类离子液体载体的 特点是在咪唑环的 C-4 或 C-5 位引入了 $\mathrm{CH}_{2} \mathrm{OH}$ linker(通

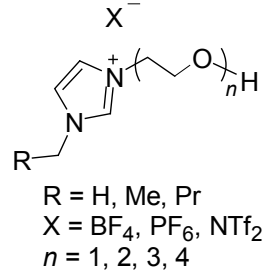

Scheme 1

常是在 C-1 位). 离子液体载体与丙烯酸反应成酯后，与 二烯发生 Diels-Alder 反应，产率较高. 同时当反应结束 后离子液体载体可以有效回收(大于 $90 \%$ ). 这种离子液 体载体的主要问题是在碱性条件下稳定性较差. 可能是 因为咪唑环上 C-2 位没有官能团化, 在碱性条件下容易 发生去质子化作用造成. 另一个不足是在反应后产物与 载体的分离需要用到剧毒的氰化钾作为酯基交换试剂 (Scheme 2).

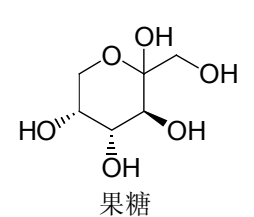

(1) $\mathrm{NH}_{3}, \mathrm{CH}_{2} \mathrm{O}, \mathrm{CuCO}_{3}$ (2) $\mathrm{BuBr}, \mathrm{KOBu}-t$, EtOH

(3) $\mathrm{Mel}, \mathrm{CH}_{2} \mathrm{Cl}_{2}$

(4) $\mathrm{NaNTf}_{2}$

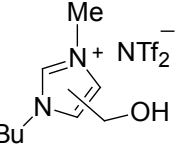

1
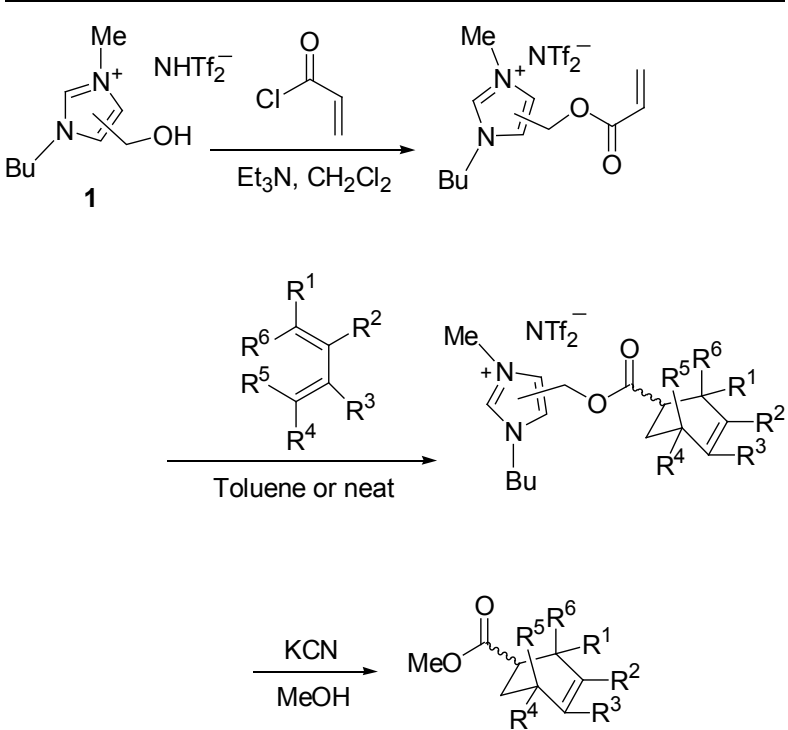

Scheme 2

2003 年, 加拿大麦吉尔大学的陈德恒小组 ${ }^{[10]}$ 发现 在 Suzuki 交叉偶联反应中使用离子液体支载的底物之 后具有了比传统液相合成更好的反应结果(Scheme 3). 在钯催化下，咪唑支载的碘苯 $\mathbf{2}$ 在水溶液中和各种芳基 嗍酸 3 发生偶联反应，高产率的得到了二芳基产物 4 . 通过加入小极性溶剂, 产物 4 可以从反应体系中沉淀出 来，从而方便的与副产物分离. 随后用碱的甲醇溶液处 理化合物 4, 除去离子液体载体的同时获得甲酯 5 . 实验 结果显示, 这个离子液体支载的 Suzuki 偶联反应和传统 
条件下的 Suzuki 反应相比, 产率更高且产物更易于纯 化. 反应活性更好的原因可能是由于离子液体支载的反 应物在水中拥有更好的溶解性, 同时咪唑片段的存在可 能对钯催化的反应有促进作用(陈德恒小组在离子液体 支载合成方面做了大量探索性工作, 例如还制备了离子 液体支载的脯氨酸用于不对称 Aldol 反应; 合成了离子 液体支载的无臭无挥发性的 Swarn 氧化试剂; 将离子液 体支载方式成功应用于多种生物大分子的合成，例如寡 肽, 寡聚脱氧核苷酸, 臭聚核苷酸, 寡糖等的合成; 并 发展出了葟聚咪唑型离子液体载体, 大大提高了可合成 臭肽的长度).
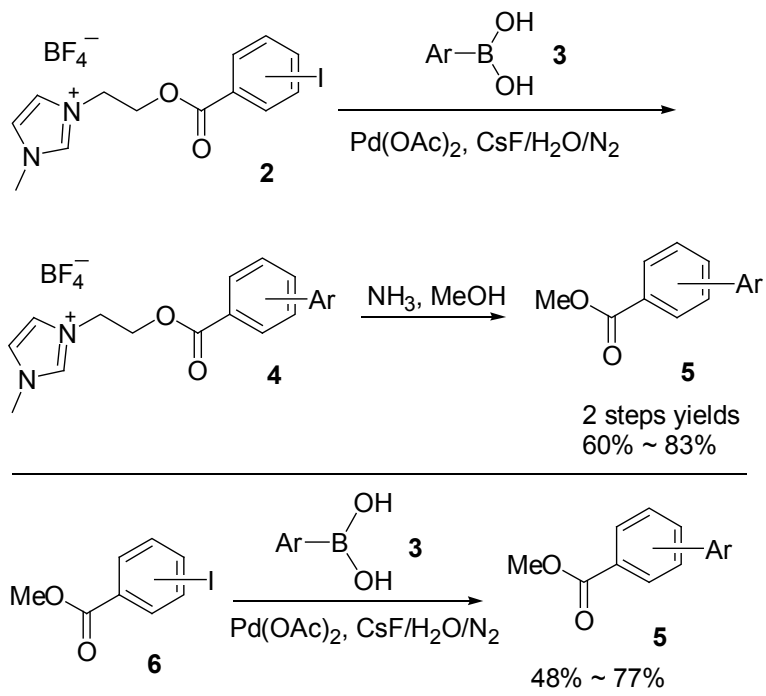

Scheme 3

2004 年, 荷兰 Organon 制药公司的 de Kort 小组 ${ }^{[1]}$ 报道了一种新型的咪唑型离子液体载体 7 , 它实际上是 AMEBA 树脂的离子液体类似物. 7 被成功应用于强效 抗血栓药物替罗非班(tirofiban)类似物 10 的多步合成. 从 8 到 9 的所有转化都是在没有有机溶剂存在下进行的, 选用了与水不互溶的离子液体 ([bmim] $\left[\mathrm{PF}_{6}\right]$ ) 作为溶剂. 在反应过程中, 采用 HPLC/MS 进行检测. 反应完成后, 剩余的反应物和副产物可以通过水和乙醚的简单淋洗 而除去, 固定在离子液体载体上的产物则留在离子液体 中, 用于下一步反应. 在多步合成过程中, 离子液体支 载的反应物在水相和有机相中均没有渗漏. 最后, 加入 酸切断离子液体载体, 通过有机溶剂萃取即可获得产物 10. 产率和纯度均可以和传统的 AMEBA 树脂固相支载 方法相媲美(Scheme 4).

2007 年, 加拿大蒙特利尔大学的 Charette 小组 ${ }^{[12]}$ 合成了四芳基磷盐支载的手性助剂 11. 在吡啶存在下, 用三氟甲磺酸酩活化 11 得到 $N$-酰亚胺吡啶盐中间体. 随后它与格氏试剂发生亲核加成反应，高区域选择性和 立体选择性的得到了 1,2-二氢吡啶 12. 通过 Adam 试剂
$\mathrm{HO}$<smiles>COc1cc(C)ccc1C=O</smiles><smiles>CCS(=O)(=O)N[R5](=O)(=O)O[Na]</smiles><smiles>COc1cc(OCCCCn2cc[n+](C)c2)ccc1C=O</smiles>

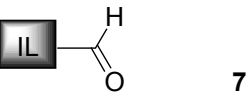

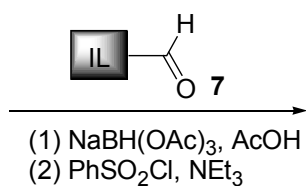

(1) $\mathrm{PhSO}_{2} \mathrm{Cl}, \mathrm{NEt}_{3}$
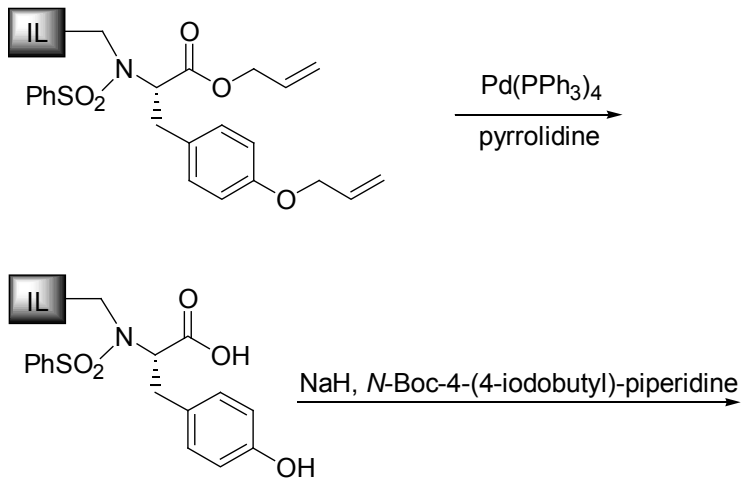

Scheme 4

催化加氢可以使哌啶衍生物和载体断开, 随后使用三溴 化硼断开畨即得到天然产物 $(-)$-毒芹碱( $13, \mathrm{R}=n-\mathrm{Pr})$ 及 其类似物. 在整个反应过程中无需使用柱层析纯化，产 率却与无支载过程相当(Charette 小组首创了四芳基磷 盐型离子液体载体, 并较广泛深入的研究了其在离子液 体支载合成中的应用. 例如四芳基磷盐试剂在 CoreyFuch 反应, Stille 偶联, Stork 还原，自由基还原环化反应 中的应用; 以及四芳基磷盐负载的常用试剂如 DCC, $\mathrm{PPh}_{3}, \mathrm{DEAD}$ 等在碳二亚胺促进的反应以及 Mitsunobu 
反应中的应用. 四芳基磷盐载体也被应用于手性小分子 及寡肽合成).
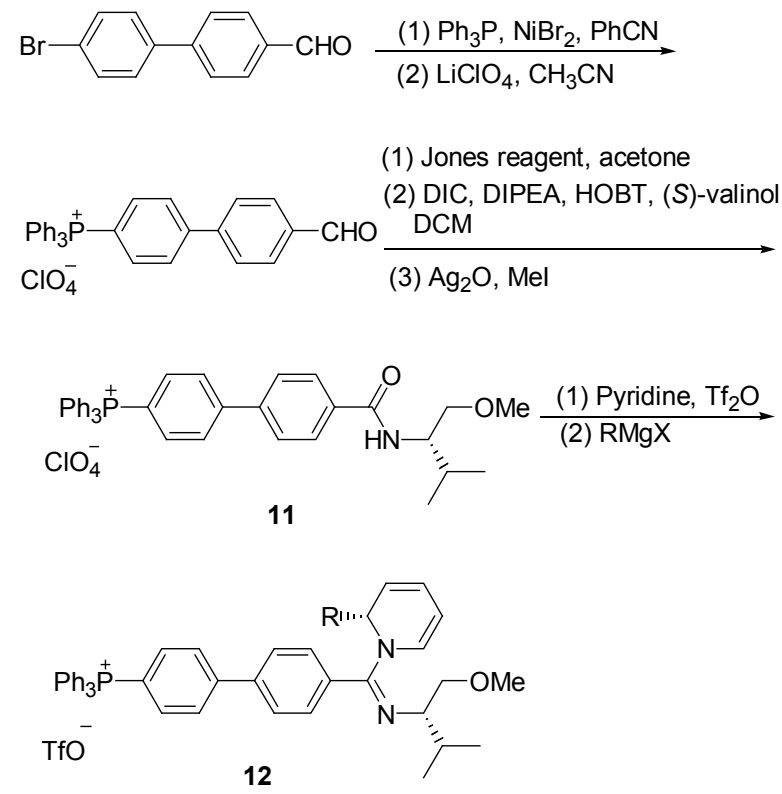

(1) $\mathrm{H}_{2}, \mathrm{PtO}_{2}, \mathrm{AcOH}$ (2) $\mathrm{BBr}_{3}, \mathrm{DCM}$

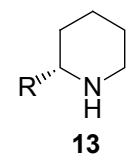

$\mathrm{R}=\mathrm{Me},(\mathrm{Z})-\mathrm{MeCH}=\mathrm{CH}, n-\mathrm{Pr}$

\section{Scheme 5}

2007 年, 浙江大学的王彦广小组 ${ }^{[13]}$ 使用离子液体 支载策略, 通过多步反应实现了顺- $\beta$-内酰胺系列化合 物的组合合成(Scheme 6). 味唑载体支载的底物 14 在 DCC/DMAP 反应条件下发生酯化反应得到离子液体支 载的醛 15. 在分子篮作干燥剂条件下, 用伯胺处理 $\mathbf{1 5}$ 得到离子液体支载的亚胺 16.16 与在三乙胺作用下由酰 氯原位生成的烯酮之间发生 Staudinger 环化加成反应, 得到离子液体支载的 $\beta$-内酰胺 17. 最后, 用三乙胺尀醇 处理使酯键断裂, 即释放出产物 $\beta$-内酰胺 18 , 纯度和产 率良好. 这一系列过程均在单一的离子液体 ([bmim]$\left.\left[\mathrm{PF}_{6}\right]\right)$ 中进行, 其中间步骤均无需使用色谱纯化, 存在 于离子液体中的离子液体载体洗涤和干燥后可以进入 下一个循环重复使用.

\section{2 离子液体支载的生物大分子合成}

离子液体载体在生物寡聚物(寡肽、寡糖、寡聚核 苷酸等)的液相合成中取得了重要的进展. 陈德恒小 组 ${ }^{[14]}$ 在 2005 年首次报道了咪唑类离子液体载体应用于 寡肽合成, 这也是离子液体支载方式首次应用于生物大 分子合成. 整个合成过程非常直观, 在均相体系中进行,
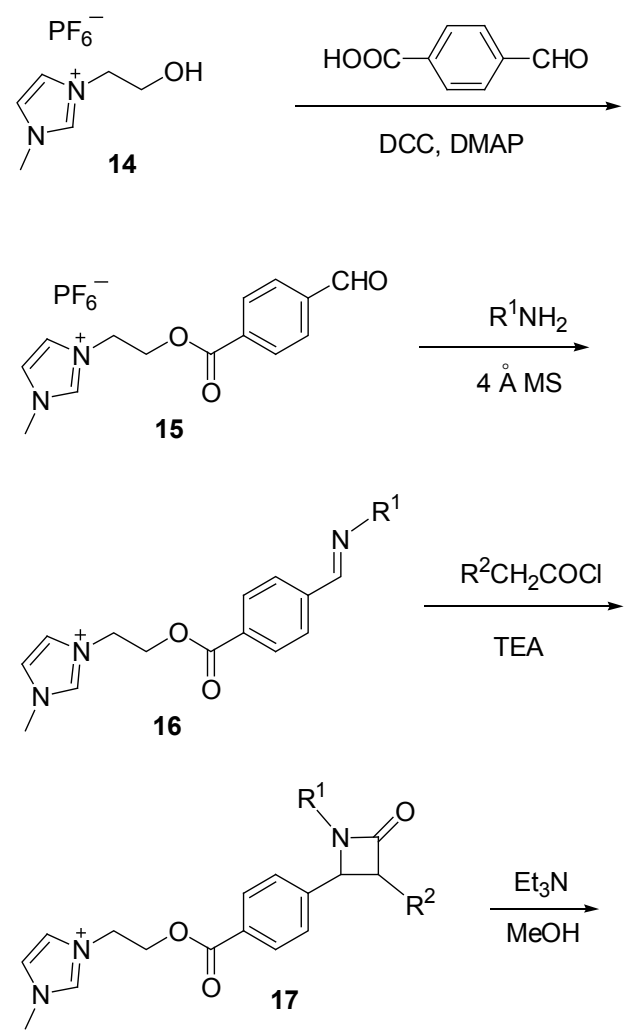<smiles>[R]C1C(=O)N([R])C1c1ccc(C(=O)OC)cc1</smiles>

Scheme 6

并且每一步都可通过常规的波谱方法进行鉴定. 在 DCC/DMAP 反应条件下，第一个氨基酸被支载到离子 液体载体上, 得到化合物 19. 然后按照常规方法使用三 氟乙酸 (TFA) 脱叔丁氧羰基 (Boc) 保护基后, 在 PyBOP/DIPEA 条件下与下一分子氨基酸偶联，循环往 复直到形成离子液体支载的五肽 20. 接下来在碱性条 件下切断酯键, 使载体与产物分离. 然后在 TFA 作用下 脱去 $\mathrm{Boc}$ 和 $t-\mathrm{Bu}$ 保护基, 即得到具有生物活性的亮氨 酸-脑啡肽. 反应总收率可达 $50 \%$, 纯度大于 $90 \%$. 在整 个合成过程中, 无需大大过量的氨基酸单体，而且在每 一步合成中都不需要重结晶或柱色谱分离. 每步中所要 做的只是使用有机溶剂和水润洗. 而且在整个反应过程 中没有观测到外消旋化或差向异构化现象. 2008 年，上 海药物研究所的柳红小组 ${ }^{[15]}$ 采用这种离子液体支载方 法合成了环状缩酚酸肽 Sansalvamide A (21), 这是一种 从海藻表面的真菌的菌丝体中分离出来的具有抗癌活 性的天然产物(Scheme 7). 


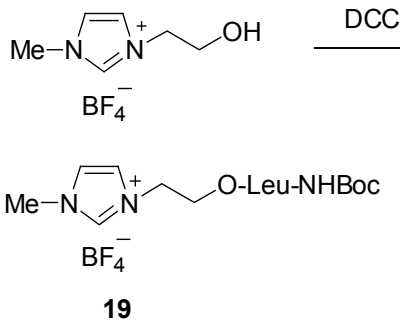

(1) TFA, $\mathrm{CH}_{2} \mathrm{Cl}_{2}$

(2) PyBOP, DIPEA, $\mathrm{CH}_{3} \mathrm{CN}$<smiles>Cc1ccc(NC(=O)OCc2ccccc2)cc1</smiles>
$\mathrm{BF}_{4}^{-}$

20
(1) $\mathrm{NaOH} / \mathrm{THF} /$ Water
HO-Leu-Phe-Gly-Gly-Tyr-H
(2) TFAVanisole
(Leu ${ }^{5}$-Enkephalin)
$>90 \%$ purity; $50 \%$ total yield

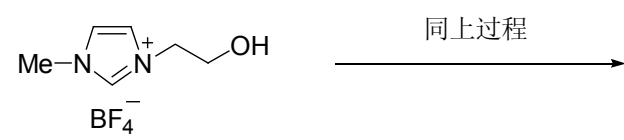

HOOC-Leu-Val-Leu-Leu-Phe- $\mathrm{NH}_{2}$

PyBOP, DIPEA, NMP

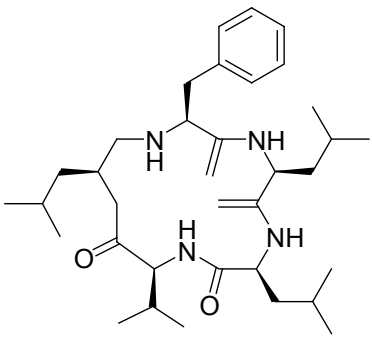

21

\section{Scheme 7}

2007 年, Charette 小组 ${ }^{[12]}$ 研发了一种以四芳基磷盐 作为离子液体载体合成肽的方法, 成功合成出具有生物 活性的飞蝗激肽. 类似于上面提到的咪唑类离子液体载 体，此类载体的优点也是在反应过程中可采用传统的检 测方法进行结构分析, 并且通过简单的沉淀过滤即可分 离得到产物. 另外, 由于最后一步载体与产物的分离过 程可以在酸性条件下进行，9-芴甲氧羰基(Fmoc)作为保 护基可以在反应过程中进行应用. 但是这些方法在用于 合成 6 个以上氨基酸的多肽时不再适用，这是由于当大 分子低聚物绑定到离子液体载体上时，随着离子液体载 体部分的比例减小, 改变了离子液体支载化合物的溶解 度. 这个问题在大多数液相支载中很常见.

为了解决上述离子液体载体无法合成超过 6 个氨基 酸的寡肽的问题, 2007 年, 陈德恒小组 ${ }^{[16]}$ 设计了一类新 的咪唑型离子低聚物作为离子液体载体. 这类化合物可 以很方便的以克的数量级进行合成(Scheme 9). 一般可 溶于水、乙醇、二甲亚砜、乙腈和硝基甲烷，在四氢呋

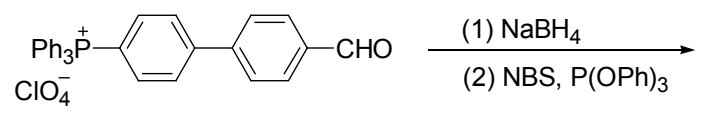

22

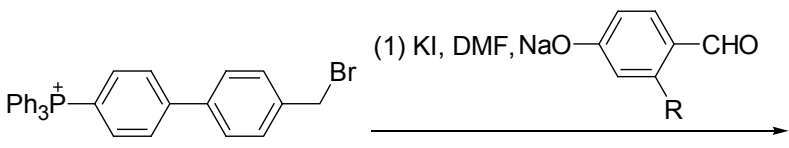

$\mathrm{ClO}_{4}^{-} \quad$ (2) $\mathrm{NaBH}_{4}, \mathrm{EtOH} / \mathrm{DCM}$

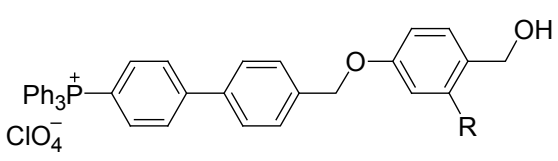

23a: $R=H \quad$ 23b: $R=O M e$

Scheme 8

喃和乙酸乙酯中溶解度较小, 不溶于烷烃、乙醚和氯仿. 使用与 Scheme 7 所示咪唑单体支载的类似方法, 这种 离子低聚物 24 可被用于寡肽的合成(Eq. 1). 由于低聚物 24 是具有多个电荷的离子液体载体, 在支载上较长的 肽链之后, 仍然具有较强的溶解度特性保持能力. 离子 液体支载寡肽合成的能力被成功提升到合成 $10 \sim 20$ 个 氨基酸长度的具有药物活性的多肽.
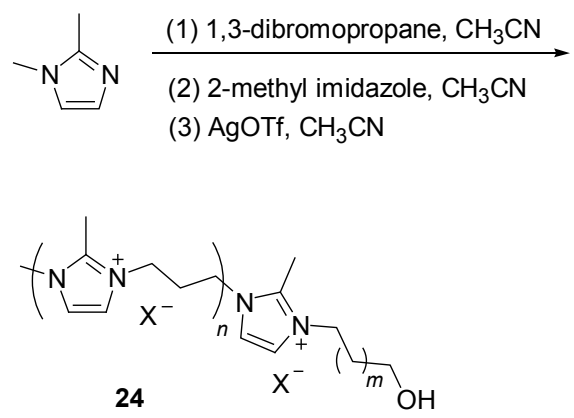

2006 年, 陈德恒小组 ${ }^{[17]}$ 将咪唑型离子液体载体成 功应用于低聚糖的合成(Scheme 9). $\beta$-硫苷 25 在 DCC/ DMAP 作用下与溴乙酸反应后，与 1-甲基咪唑反应成 盐, 再和四氟砋酸钠进行反离子交换, 得到离子液体支 载的单糖 26. 化合物 26 用 $m-\mathrm{CPBA}$ 氧化得到亚砜 27 作 为糖基供体. 25 和 27 发生亚砜糖基化反应，即得到离子 液体支载二糖 28. 重复相同的过程，就可以离子液体支 载的三糖 29. 在碳酸铯/甲醇体系中, 离子液体载体被 切断后得到三糖 30. 这种方法提供了一条在整个合成 过程中无需使用色谱纯化的寡糖构造方案. 中间产物结 构可以用 NMR, MS 和其他传统技术进行表征. 此外, 采用离子液体支载合成时的立体选择性与传统的液相 合成一致，均生成 $\beta$-构型产物.

同期王彦广小组 ${ }^{[18]}$ 也报道了离子液体支载的寡糖 合成. 在他们的例子中, 离子液体载体被链接到了糖 31 

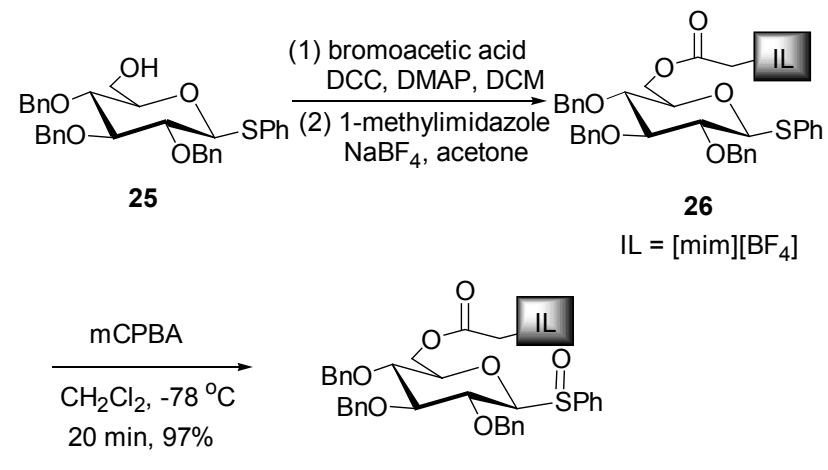

27
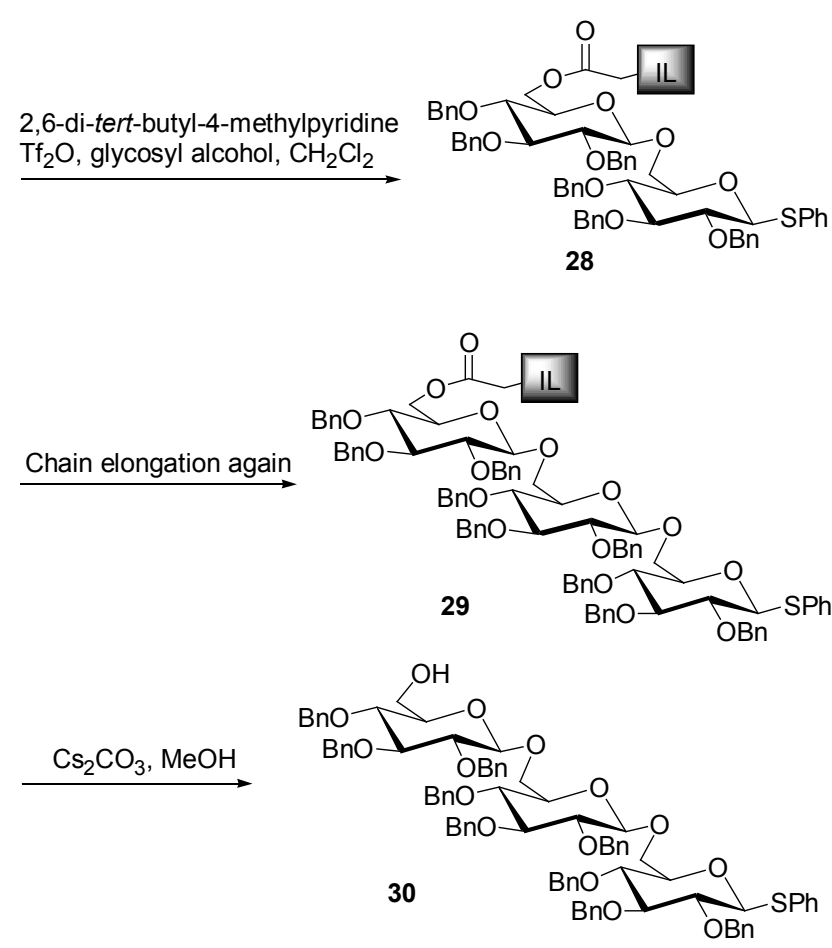

Scheme 9

的 4 号位. 而 $\alpha$-三氯乙酰亚胺酯活化的糖合成子 32 被 用作糖基供体连接到 6 号位去延伸糖链(Scheme 10).

最近, 类似的方法已被用来合成含有 8 个单糖单元 的 $\alpha$-辛甘露糖苷 ${ }^{[19]}$.

虽然在多种生物寡聚物的合成中都取得了相当的 进展，离子液体支载的生物大分子合成仍然面临挑战: 是否能够合成具有更高分子重量的聚合物?

\section{3 离子液体支载的催化剂}

出于环境保护方面的需求以及经济方面的考虑, 发 展可循环和可再生的催化剂, 特别是贵重过渡金属催化 剂是现代合成化学的一个重要研究主题. 离子液体支载 的催化剂, 由于可以方便的与底物和产物分离, 回收并 再次使用而引起了人们的兴趣. 目前对离子液体支载的 金属复合物的研究主要集中于: (1)钉 $(\mathrm{Ru})$ 和铑 $(\mathrm{Rh})$ 催化

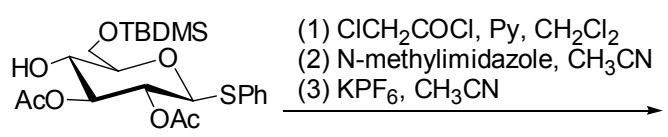

31
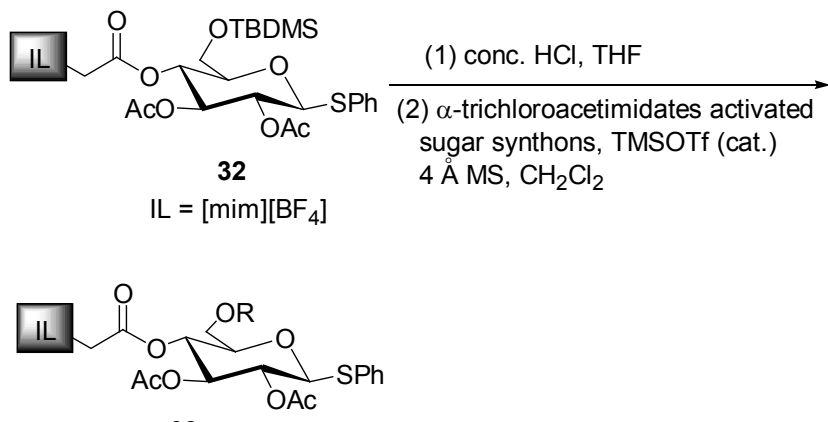

33

$\mathrm{R}=\mathrm{Glu}, \mathrm{Rha}, \mathrm{Xyl}, \mathrm{Man}$, Fuc, Mal

\section{Scheme 10}

的烯烃复分解反应、氢化反应和氢甲酰化反应; (2)钯 $(\mathrm{Pd})$ 催化的交叉偶联反应，特别是 Heck 反应和 Suzuki 偶联反应. 离子液体支载的配体用于铜 $(\mathrm{Cu})$, 铁 $(\mathrm{Fe})$, 镍 $(\mathrm{Ni})$, 镇 $(\mathrm{Mn})$, 钛 $(\mathrm{Ti})$, 钒 $(\mathrm{V})$ 等金属催化的反应也有零 星报道.

由于钓和铑在催化有机反应中应用广泛但价格高 昂，离子液体支载的钓和铑金属配合物是本领域的一个 研究热点. 由于反应条件温和、官能团耐受性强、反应 活性高、前催化剂稳定以及起始原料简单易得等诸多优 点, 烯烃复分解反应是生成 $\mathrm{C}=\mathrm{C}$ 键的重要方法. $\mathrm{Grubb}$ 钓催化剂在室温离子液体中可以有效的催化烯烃复分 解反应，但是催化剂在进一步的循环使用中会迅速失 活. 原因在于后处理时催化体系分解或者在产物萃取步 骤损失到有机溶剂中. 为了克服这个缺点, 法国雷恩国 立高等化学学院的 Guillemin 小组 ${ }^{[20]}$ 和美国北伊利诺斯 州大学的 Yao 小组 ${ }^{[21]}$ 分别于 2003 年首次报道了咪唑离 子液体支载的钓催化剂用于烯烃复分解反应，此后两个 小组分别对此进行了较为深入的研究. 由 Guillemin 和 Yao 领导的两个研究小组, 独立的设计了几乎相同的咪 唑离子液体支载配合物来催化 Hoveyda-Grubbs 反应. 这两种催化剂唯一的区别在于催化剂的 linker 长度不同 (2: Guillemin, 3: Yao)(Eq. 2). 这些离子液体支载催化剂 通过简单的合成过程即可制备. 以催化剂 35 的合成为 例, 由 38 和 1-氯-4-碘丁烷之间脱去一分子碘化氢合成 39.39 和咪唑在 $\mathrm{NaOH}$ 作用下得到 40.40 与碘甲烷反应 后和 $\mathrm{AgPF}_{6}$ 发生阴离子交换即生成咪唑型离子液体载 体 41. 用原始的 Grubbs 催化剂处理 41 即可得到离子液 体支载催化剂 35 (Scheme 11). 这两种离子液体支载的 


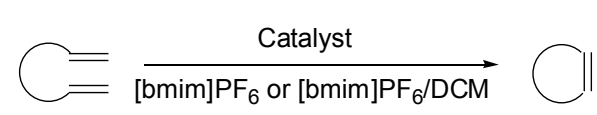

(2)

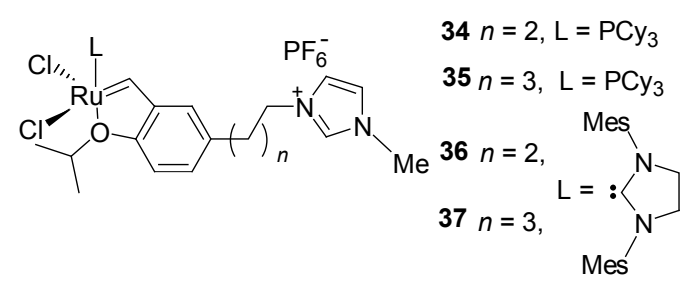

催化剂在离子液体或是两相体系(离子液体/二氯甲烷) 中均表现出较高的反应活性和显著的可回收性. 催化剂 34 可以稳定存在于离子液体 $\left([\mathrm{bmim}]\left[\mathrm{PF}_{4}\right]\right)$ 中几个月而 不失活性. 离子液体支载的钉催化剂 34 和 35 在循环使 用 10 次后, 活性依然很高, 在烯烃关环复分解反应中转 化率可以超过 90\%. 然而, Guillemin 提到, 含氧双烯作 为底物时, 离子液体支载催化剂的循环能力大大降低 (只能循环 $1 \sim 2$ 次), 原因在于底物中的氧原子可以造成 催化剂体系的分解.

两年后, Guillemin 和 Yao 等 ${ }^{[22]}$ 分别报道了他们对其 离子液体支载催化剂的改进. $N$-杂环卡宾钉配合物 36 和 37 更适合于在较剧烈条件下催化活性较低的双烯底物 进行关环复分解反应. 二、三、四取代的双烯以及炔稀 都取得了良好的效果. 在含氧和含硫体系中, 化合物 36 也可以进行很好的多次循环使用, 并且保持了良好的可 回收性. 此外, 离子液体/甲苯两相体系的使用, 进一步 提高了催化剂 36 的回收率, 极大的降低了钉在最终产 品中的残留水平.

在 $2006 \sim 2007$ 年, 一系列用吡定离子液体支载的 钓催化剂也被报道可用于烯烃复分解反应. 但是这些催 化剂的回收循环使用效果并不理想. 其中最优的化合物 只可循环使用 6 次 ${ }^{[23]}$.

钯催化偶联反应是碳碳键构建的强有力工具. 许多 钯催化剂都使用磷配体, 然而离子液体支载的磷配体通 常容易被氧化生成磷氧化合物, 不易回收循环使用. 于 是研究者的兴趣主要集中于发展离子液体支载的非磷 配体. 2004 至 2007 年美国爱达荷大学的 Shreeve 小组 ${ }^{[24]}$ 对这一课题进行了较为系统的研究, 发展了一系列咪唑 离子液体支载的氮配体, 并将其与氯化钯反应制备了一 系列钯复合物 42, 43 和 44 (Scheme 12 和 Eq. 3), 在 Heck, Suzuki 和 Sonogashira 反应中都取得了很好的效果. 在 Suzuki 偶联和 Heck 反应中, 离子液体载体既作为钯催 化剂的配体, 同时又作为离子液体充当溶剂去发生交叉 偶联反应(Eq. 3). 而催化剂 44 在 Sonogashira 反应中活 性良好. 产物可以通过乙醚萃取而很容易的从反应体系 中分离出来. 然后催化剂/离子液体体系可以用水洗
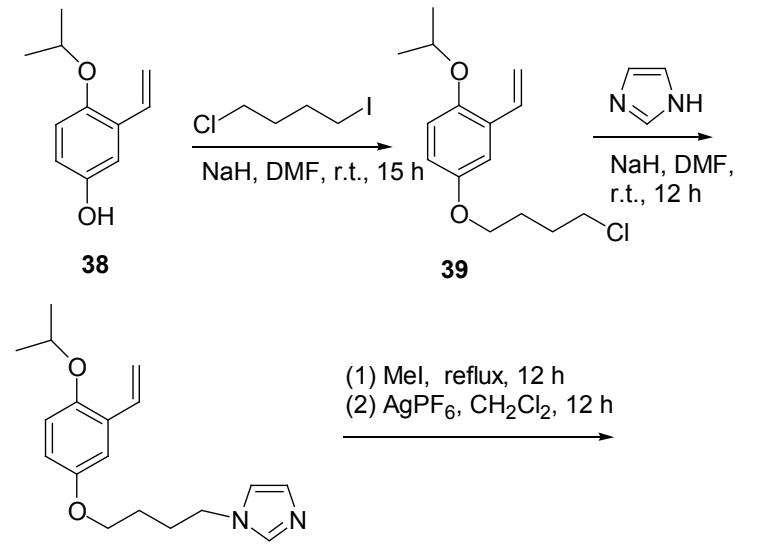

(1) Mel, reflux, $12 \mathrm{~h}$

(2) $\mathrm{AgPF}_{6}, \mathrm{CH}_{2} \mathrm{Cl}_{2}, 12 \mathrm{~h}$
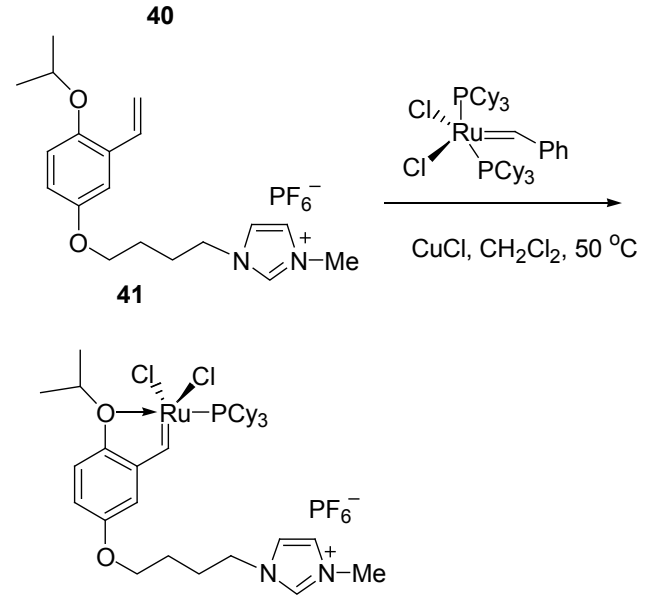

35

Scheme 11
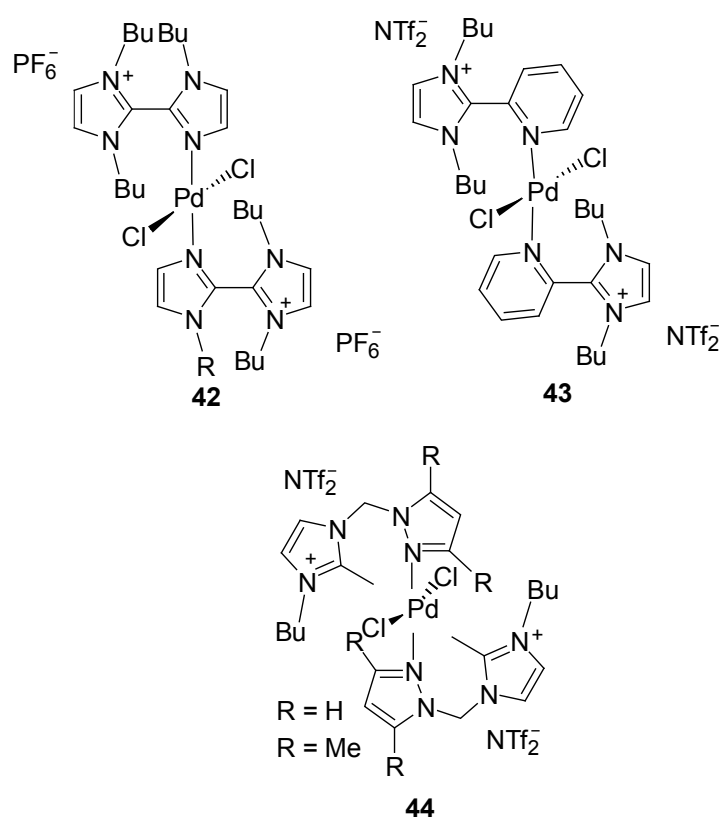

\section{Scheme 12}

去钠盐并真空干燥后进行下一循环使用, 萃取过程中钯 的浸出量非常小, 催化剂可以重复使用 9 15 次. 


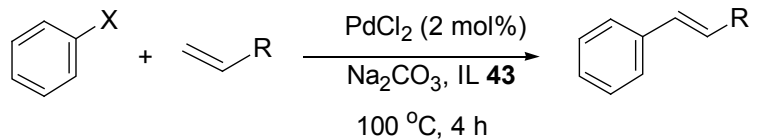

2007 年, 华东师范大学的刘晔小组 ${ }^{[25]}$ 报道了咪唑 离子液体支载的二醇配体 45 可以作为非膦配体用于钯 催化的 Heck 反应(Scheme 13). 催化剂表现出良好的稳 定性, 回收循环使用 10 余次而活性不减, 在碘苯或溴苯 类化合物与末端丙烯酸酯的偶联反应中钯无明显浸出.

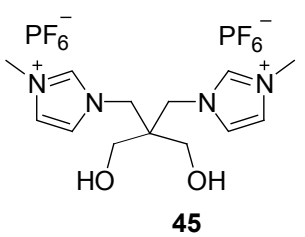

\section{Scheme 13}

除了以上咪唑支载的配合物以外, 鎓盐同样也可以 用做离子液体载体(Scheme 14). 2009 年, 意大利博洛尼 亚大学的 Lombardo 小组 ${ }^{[26]}$ 设计出一种在空气中稳定且 易于操作的三乙胺支载的二苯基磷钯化合物 46 . 用 46 做催化剂, 芳基溴化物和芳基硣酸在吡咯烷鎓盐离子液 体中进行 Suzuki-Miyayra 反应可以得到很好的产率. 反 应没有表现出在通常钯催化的反应中生成钯黑的不良 趋势. 提取产物后, 包含催化剂的离子液体可以方便的 回收循环使用 6 次，而催化活性基本没有损失.

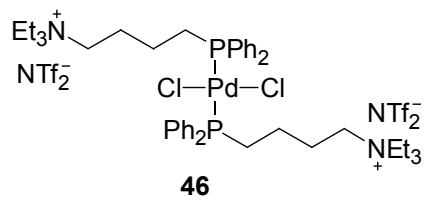

\section{Scheme 14}

除了离子液体支载的金属催化剂体系, 有机小分子 也可以被嫁接到离子液体载体上. 目前离子液体支载的 有机小分子催化剂主要应用于不对称 Aldol 反应和 Michael 加成反应. 2006 年, 陈德恒小组 ${ }^{[27]}$ 首次报道了 咪唑离子液体支载的脯氨酸应用于不对称 Aldol 反应. 随后, Lombardo 小组 ${ }^{[28]}$ 合成了大量类似物, 并评估了其 在不对称 Aldol 反应中的效果, 找到了一些很好的体系. 同期我国南开大学的程津培小组 ${ }^{[29]}$ 、浙江工业大学的徐 振元小组 ${ }^{[30]}$ 、兰州大学的梁永民小组 ${ }^{[31]}$ 都先后报道了咪 唑离子液体支载的吡咯烷催化剂在不对称 Michael 加成 反应中的应用(程津培小组还研究了离子液体支载的不 对称 Aldol 反应 ${ }^{[32]}$ 、Baylis-Hillman 反应 ${ }^{[33]}$ 、以及离子 液体支载的奎宁类化合物 ${ }^{[34]}$.

\section{4 离子液体支载的化学计量反应试剂}

离子液体支载的试剂与离子液体支载的催化剂不 同, 在反应中需要以化学计量或更高剂量使用, 并且在 反应过程中本身发生化学转化. 在引入离子载体后, 支 载化试剂及其转化产物可以通过加入较小极性的溶剂 使之沉淀, 从而方便的和底物及产物分离. 为了使离子 液体支载的试剂可以真正具有实用价值并在合成中广 泛使用，离子液体支载的试剂需要具有以下特点：(1)容 易制备; (2)试剂及其转化产物容易从反应混合物中回 收; (3)转化产物能够方便的再生为试剂本身, 再次使用.

离子液体支载的试剂研究目前主要集中于Wittig 类 型的反应和 Stille 类型的碳碳键构建反应.

Wittig 反应和它的变体使用三苯基膦作为媒介将羰 基化合物转化成烯烃，在有机合成中的应用非常广泛. 如何将产物和反应的副产物三苯氧膦 $\left(\mathrm{Ph}_{3} \mathrm{PO}\right)$ 分开是一 个长期困扰合成化学家的问题, 通常需要繁琐的柱色谱 分离或是多次重结晶才可以彻底分开. 为了解决这个问 题，在聚合物支载三苯基膦和氟支载三苯基膦两个方向 都做了大量的研究. 2006 年, Charette 小组 ${ }^{[35]}$ 报道了用四 苯基膦盐(48)支载的三苯基膦试剂，它可以成功应用于 Corey-Fuchs 反应. 四苯基膦盐 48 的合成是通过间一溴苯 二苯基磷(47)在镍催化下和三苯基膦发生反应，随后进 行阴离子交换而得到的(Scheme 15). 高氯酸盐 48 化学 性质稳定, 在含氯溶剂中具有良好的溶解性, 在加入小 极性溶剂后可以完全沉淀出来. 而且在沉淀过程中不会 包夹有机化合物. 48 和醛及四溴化碳在 Corey-Fuchs 反 应条件下生成 $\alpha, \alpha$-二溴烯烃 50 和氧化磷 49. 通过简单 的沉淀和过滤就可以将 49 和 50 分开, 并通过核磁共振 磷谱证实了产物中无副产物 49 存在. 反应收率和传统
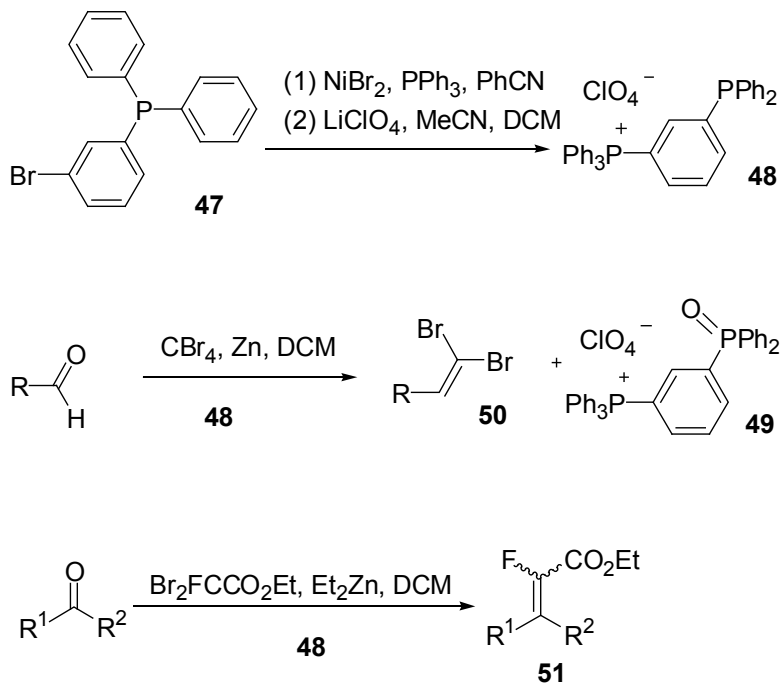

\section{Scheme 15}


的三苯基膦反应相同. 氧化磷 49 可以用三氯硅烷和二 甲苯胺还原再生试剂 48. 离子液体支载的三苯基膦 48 在二甲基锌的促进下也可以将羰基化合物转变为 $\alpha$-氨$\alpha, \beta$-不饱和酯类化合物 51. 同样, 离子液体支载膦的化 合物 49 和氧化磷 50 可以方便的通过沉淀及过滤的方法 和烯烃分开. 但这种四苯基膦盐支载的三苯基膦试剂并 没有被成功应用于经典 Witting 反应或是 Witting-Horner 反应的报道.

2008 年, 陈德恒小组 ${ }^{[36]}$ 报道了以 TPPMS 作为磺酸 根阴离子支载的三苯基磷在 Witting 反应中的应用 (Scheme 16). TPPMS 52 可以和多种卤代烃反应生成两 性膦盐 53. 53 和醛在碱性条件下发生反应生成烯烃 55 和氧化膦 (54, TPPMSO). 多余的试剂和副产物 TPPMSO (54)可以通过加入小极性溶剂(例如乙醚)而简 便地沉淀出来, 从而与主产物烯烃分离. 由此获得的烯 烃通过核磁共振氢谱检测纯度很好, 并通过核磁共振磷 谱证实产物中无磷信号, 无需使用柱色谱进行分离纯 化. TPPMSO (54) 可以用三氯硅烷和三苯基膦处理而重 新生成 TPPMS. 应用此反应方法合成甲氧基保护的天 然产物白藜芦醇, 产率可高达 90\%. 当甾代烃换成溴乙 酸酯时, 可以采用一锅法直接合成 $\alpha, \beta$-不饱和酯.
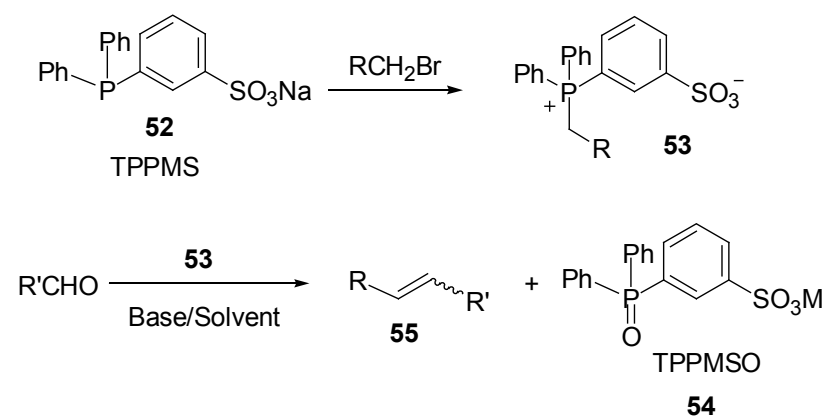

\section{Scheme 16}

和其它支载试剂相比, 离子支载磷试剂 48 和 52 对 于 Wittig 反应非常实用. 由于大多数支载试剂合成过程 繁琐，只见文献报道而并未商品化销售，支载试剂在有 机合成和产物纯化中的广泛适用性遭到质疑. 离子液体 支载试剂 48 可以利用试剂 47 通过一步反应即可制备. 而化合物 52 直接是商品化的试剂, 目前主要在工业生 产中用作酰基化反应中铑催化剂的配体. 其次, 由于离 子液体支载的 Wittig 试剂的相对分子质量和相关的聚合 物载体相比小很多, 所以离子液体支载的能力比聚合物 支载要高出很多. 与含氟化合物支载的 Wittig 试剂相比, 离子液体支载的 Wittig 反应可以在一系列常规溶剂中进 行而无需全氟溶剂. 所以离子支载试剂所提供的实际优 势是聚合物支载和氟化物支载的 Wittig 试剂所不及的.
48 和 52 的区别在于, 48 使用的是阳离子支载, 而 52 用 的是阴离子支载. 对于它们的区别虽然还没有做直接的 比较, 但似乎电荷性质会影响它们作为 Wittig 反应中间 体的能力. 52 在经典的 Wittig 反应中非常有效, 但不适 用于 Corey-Funchs 反应. 而 48 在 Corey-Funchs 反应中 非常有效, 然而到目前为止, 用 48 实现经典 Wittig 反应 的研究还未见报道.

2007 年, 法国勒芒大学的 Legoupy 小组 ${ }^{[37]}$ 报道了离 子液体支载的锡试剂可成功应用于 Stille 交叉偶联反 应. Stille 偶联是一种经典的 $\mathrm{C}-\mathrm{C}$ 键构建方法. 但是, 有机锡化合物也有其致命的弱点, 即毒性很强且难以和 产物彻底分离. 因此，在医药品的合成应用中大受限制. 在离子液体 $[\mathrm{Bmim}]\left[\mathrm{PF}_{4}\right]$ 中, 或无溶剂条件下, 离子液体 支载的锡试剂 59 和碘苯可以顺利发生交叉偶联反应得 到联苯 61, 产率高达 90\%以上(Scheme 17). 反应结束 后, 联苯 61 和其它原料以及副产物可通过正戊烷这样 的小极性有机溶剂萃取出来, 剩余的钯催化剂和离子液 体支载的有机锡卤化物 60 则会留在离子液体中. 副产 物离子液体支载的有机锡卤化物可以通过简单的方法 再生离子液体支载的锡试剂 59. 再生的试剂可以再次 和碘苯发生偶联，但活性有所下降，产物 61 的收率降至 $72 \%$.

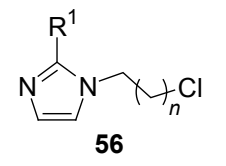

LDA, $\mathrm{Sn}(n-\mathrm{Bu})_{2} \mathrm{PhH}(\mathbf{5 7})$, THF

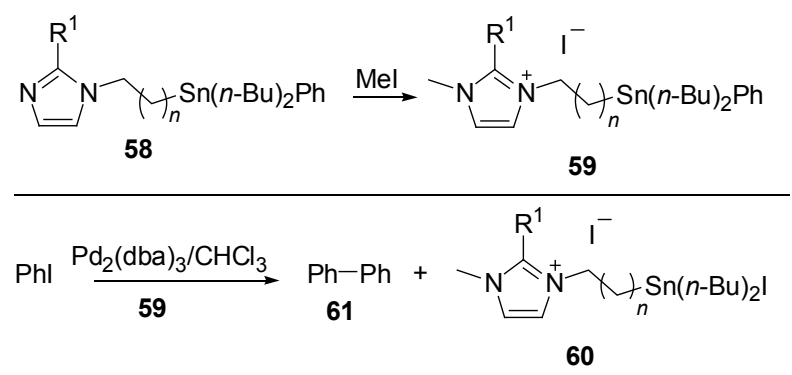

Scheme 17

2007 年, Charette 小组 ${ }^{[38]}$ 报道了一种效果更好的离 子液体支载锡试剂. 他们首先通过三步反应制备了四芳 基膦盐支载的锡试剂 62.62 和各种芳基溴化物在钯催化 剂存在下, 以二氧六环作溶剂, 高产率得到了 Stille 反 应产物(Eq. 4), 产率可比得上用三正丁基锡在同样条件 下反应的结果. 反应结束后，加入正己烷即可使产物从 体系中分离出来, 过滤即可除去离子液体支载的锡溴化 物，滤液蒸干即可得到不含锡的偶联产物. 同样，反应 产生的锡溴化物可以回收，用乙烯基溴化镁处理后可再 
生离子液体支载的乙烯基锡试剂 62.

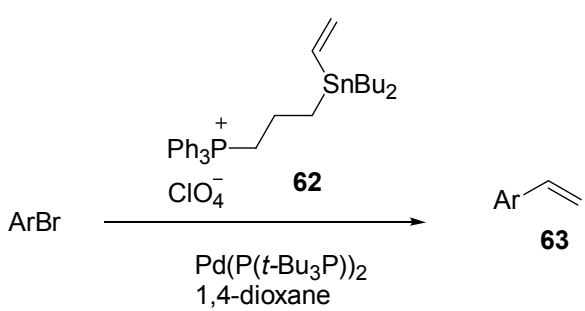

离子液体支载的试剂也已经被应用于多种官能团 转化反应, 例如氧化、还原、碳二亚胺促进的反应以及 Mitsunobu 反应等.

\section{5 展望}

在 21 世纪的第一个十年里, 离子液体载体在有机 合成中的应用取得了初步的成果, 在支载反应底物、催 化剂、化学计量反应试剂方面都取得了不同程度的进展, 开始引起越来越多有机化学工作者的兴趣. 与其它类型 的支载合成(例如不溶性高分子支载的固相合成、可溶 性高分子支载的液相合成、以及氟化物支载合成)相比, 离子液体支载合成具有均相反应条件、上载率高、合成 简单、易于放大、反应可在常规溶剂中顺利进行、反应 中间体便于利用传统波谱手段例如核磁共振、质谱等进 行鉴定等特点. 离子液体支载的试剂在具有母体试剂类 似反应性的同时, 由于离子化物质的溶解特性, 使后处 理步骤大大简化, 通常采用沉淀、萃取、过滤、洗涤等 方式即可得到产物. 在多步合成中, 好的离子液体载体 的应用可以大大简化反应处理步骤, 提高效率与产率; 并且离子液体支载使催化剂的回收、循环使用, 以及化 学当量试剂的回收、再生成为可能. 然而作为一个新兴 的领域, 目前可以应用的离子液体载体类型还非常有 限, 主要局限于应用咪唑阳离子型和吡啶阳离子型载 体, 另有少量应用鈆盐和磷盐作为载体的报道. 所以探 索发展原料简单易得, 合成步骤简短; 易于回收, 易于 再生; 具有实用价值的离子液体载体可以拓宽离子液体 支载合成的应用范围, 将相关领域的研究引向深入. 另 外, 目前离子液体支载的生物大分子合成虽然取得了一 定进展, 但还处于起步阶段. 例如离子液体支载的寡肽 合成长度一般局限在 5 9 个氨基酸, 寡糖的合成长度 则局限在 $4 \sim 8$ 个单糖. 应用新型离子液体载体增大可 合成生物寡聚物的长度, 发展更有效的生物寡聚物合成 路线, 得到可以进行规模化生产的离子液体支载生物塞 聚物合成工艺, 将是非常有意义的研究领域. 而目前离 子液体支载的有机小分子合成实例都还较为简单; 离子 液体支载的催化剂和化学计量反应试剂研究也还局限
在有限的几个反应类型当中. 但它们已经展现出一些优 良特点, 表明了继续拓宽和深入研究的必要性.

\section{References}

[1] (a) Merrifield, R. B. J. Am. Chem. Soc. 1963, 85, 2149.

(b) Solinas A.; Taddei, M. Synthesis 2007, 2409.

(c) Lu, J.; Toy, P. H. Chem. Rev. 2009, 109, 815.

[2] (a) Bergbreiter D. E.; Sung, S. D. Adv. Synth. Catal. 2006, 348, 1352 .

(b) Gravert, D. J.; Janda, K. D. Chem. Rev. 1997, 99, 489.

[3] (a) Studer, A.; Hadida, S.; Ferritto, R.; Kim, S. Y.; Jeger, P.; Wipf, P.; Curran, D. P. Science 1997, 275, 823.

(b) Horvath, I. T. Acc. Chem. Res. 1998, 31, 641.

[4] Wilkes, J. S.; Zaworotko, M. J. J. Chem. Soc., Chem. Commun. $1992,13,965$.

[5] Davis, J. H. Jr.; Forrester, K. J. Tetrahedron Lett. 1999, 40, 1621.

[6] Fraga-Dubreuil, J.; Bazureau, J. P. Tetrahedron Lett. 2001, 42, 6097.

[7] (a) Huo, C.; Chan, T. H. Chem. Soc. Rev. 2010, 39, 2977. (b) Miao, W.; Chan, T. H. Acc. Chem. Res. 2006, 39, 897. (c) Pereira, M. M. A. Curr. Org. Chem. 2012, 16, 1680.

[8] (a) Fraga-Dubreuil, J.; Bazureau, J. P. Tetrahedron Lett. 2001, 42, 6097.

(b) Fraga-Dubreuil, J.; Bazureau, J. P. Tetrahedron 2003, 59, 6121. (c) Fraga-Dubreuil, J.; Famelart, M.-H.; Bazureau, J. P. Org. Process Res. Dev. 2002, 6, 374.

(d) Hakkou, H.; Eynde, J. J.; Hamelin, J.; Bazureau, J. P. Tetrahedron 2004, 60, 3745.

(e) Legeay, J. C.; Eynde, J. J.; Bazureau, J. P. Tetrahedron 2005, $61,12386$.

(f) Legeay, J. C.; Goujon, J. Y.; Eynde, J. J.; Toupet, L.; Bazureau, J. P. J. Comb. Chem. 2006, 8, 829.

(g) Debdab, M.; Mongin, F.; Bazureau, J. P. Synthesis 2006, 4046.

[9] Handy, S. T.; Okello, M. Tetrahedron Lett. 2003, 44, 8399.

[10] Miao, W.; Chan, T. H. Org. Lett. 2003, 5, 5003.

[11] de Kort, M.; Tuin, A. W.; Kuiper, S.; Overkleeft, H. S.; vander Marel, G. A.; Buijsman, R. C. Tetrahedron Lett. 2004, 45, 2171.

[12] Stazi, F.; Marcoux, D.; Poupon, J.-C.; Latassa, D.; Charette, A. B. Angew. Chem., Int. Ed. 2007, 46, 5011.

[13] Tao, X.-L.; Lei, M.; Wang, Y.-G. Tetrahedron Lett. 2007, 48, 5143-5146.

[14] Miao, W.; Chan, T. H. J. Org. Chem. 2005, 70, 3251.

[15] Chen, L.; Zheng, M.; Zhou, Y.; Liu, H.; Jiang, H. Synth. Commun. 2008, 38, 239.

[16] He, X.; Chan, T. H. Org. Lett. 2007, 9, 2681.

[17] He, X.; Chan, T. H. Synthesis 2006, 1645.

[18] Huang, J. Y.; Lei, M.; Wang, Y.-G. Tetrahedron Lett. 2006, 47, 3047.

[19] Yerneni, C. K.; Pathak, V. A.; Pathak, K. J. Org. Chem. 2009, 74, 6307.

[20] Audic, N.; Clavier, H.; Mauduit, M.; Guillemin, J. C. J. Am. Chem. Soc. 2003, 125, 9248.

[21] Yao, Q.; Zhang, Y. Angew. Chem., Int. Ed. 2003, 42, 3395.

[22] (a) Clavier, H.; Audic, N.; Guillemin, J.-C.; Mauduit, M. J. Organomet. Chem. 2005, 690, 3585. (b) Yao, Q.; Sheets, M. J. Organomet. Chem. 2005, 690, 3577.

[23] Rix, D.; Caijo, F.; Laurent, I.; Gulajski, L.; Grela, K.; Mauduit, M. Chem. Commun. 2007, 3771.

[24] Wang, R.; Piekarski, M. M.; Shreeve, J. N. M. Org. Biomol. Chem. 2006, 4, 1878. 
[25] Cai, Y. Q.; Lu, Y.; Liu, Y.; Gao, G. H. Catal. Lett. 2007, 119, 154.

[26] Lombardo, M.; Chiarucci, M.; Trombini, C. Green Chem. 2009, 11, 574.

[27] Miao, W.; Chan, T. H. Adv. Synth. Catal. 2006, 348, 1711.

[28] (a) Lombardo, M.; Pasi, F.; Easwar, S.; Trombini, C. Adv. Synth. Catal. 2007, 349, 2061.

(b) Lombardo, M.; Easwar, S.; De Marco, A.; Pasi, F.; Trombini, C. Org. Biomol. Chem. 2008, 6, 4224.

(c) Lombardo, M.; Easwar, S.; Pasi, F.; Trombini, C. Adv. Synth. Catal. 2009, 351, 276.

[29] Luo, S.; Mi, X.; Zhang, L.; Liu, S.; Xu, H.; Cheng, J.-P. Angew. Chem., Int. Ed. 2006, 45, 3093.

[30] Xu, D.; Luo, S.; Yue, H.; Wang, L.; Liu, Y.; Xu, Z. Synlett 2006, 2569.
[31] Wu, L.-Y.; Yan, Z.-Y.; Xie, Y.-X.; Niu, Y.-N.; Liang, Y.-M. Tetrahedron: Asymmetry 2007, 18, 2086.

[32] Luo, S.; Mi, X.; Zhang, L.; Liu, S.; Xu, H.; Cheng, J.-P. Tetrahedron 2007, 63, 1923.

[33] Mi, X.; Luo, S.; Cheng, J.-P. J. Org. Chem. 2005, 70, 2338.

[34] Mi, X.; Luo, S.; Xu, H.; Zhang, L.; Cheng, J.-P. Tetrahedron 2006, 62, 2537.

[35] Poupon, J.-C.; Boezio, A. A.; Charette, A. B. Angew. Chem., Int. Ed. 2006, 45, 1415.

[36] Huo, C.; He, X.; Chan, T. H. J. Org. Chem. 2008, 73, 8583.

[37] Vitz, J.; Mac, D. H.; Legoupy, S. Green Chem. 2007, 9, 431.

[38] Poupon, J.-C.; Marcoux, D.; Cloarec, J.-M.; Charette, A. B. Org. Lett. 2007, 9, 3591. 